\title{
Nature des radiolésions induites dans l'acide désoxyribonucléique et leur réparation $\left(^{*}\right)$
}

\author{
E. MOUSTACCHI $(* *)$
}

(Manuscrit reçu le 24 février 1976)

\begin{abstract}
RÉSUMÉ
La nature des différentes lésions provoquées par les rayonnements ionisants au niveau de l'ADN est décrite. Il s'agit de l'induction de ruptures d'un brin ou des deux brins de la chaîne d'ADN et de modifications des bases. Les principaux processus enzymatiques de réparations de ces altérations du matériel génétique sont rappelés.
\end{abstract}

\begin{abstract}
The nature of the different damages induced by ionizing radiations in DNA is described. The main lesions are single strand breaks, double strands breaks and base modifications. The principal enzymatic repair systems are recalled.
\end{abstract}

Il est très généralement admis que la principale cible cellulaire endommagée par les radiations est constituée par l'acide désoxyribonucléique et que ce sont les dommages infligés à ce composant fondamental du noyau qui sont responsables de la mort cellulaire et des effets génétiques tels que l'induction de mutations ou l'induction d'aberrations chromosomiques. Je ne reviendrai pas sur les arguments expérimentaux qui font admettre cette notion, mais ce fut la tâche principale des radiobiologistes jusqu'aux années 60 . Ceci n'exclut pas qu'il y ait des lésions autres que celles se situant au niveau de l'ADN qui soient responsables de la mort cellulaire, mais disons que cette notion a été l'une des plus fructueuses sur le plan expérimental. La décennie

(*) Conférence présentée au Séminaire de Radiobiologie, organisé par la Société française de Radioprotection, à Fontenay-aux-Roses, les 29-30 avril 1975.

(**) Fondation Curie, Institut du Radium, Faculté des Sciences, Bât. 110, 91405 Orsay.

IOPROTECTION, VOL. $11-\mathrm{N}^{\circ} 4$ 
suivante a vu l'éclosion d'un nombre considérable de travaux s'attachant à analyser la nature chimique des lésions produites au niveau des acides nucléiques. Je vais tâcher de faire le bilan très bref de ces travaux concernant l'analyse fine des lésions effectuées au niveau de l'ADN.

Considérons un modèle simplifié où l'on voit la double hélice avec les ponts hydrogène qui relient les plateaux des bases; en ce qui concerne ces plateaux de bases, vous savez que la thymine est toujours associée à l'adénine et la cytosine à la guanine. On a aussi un désoxyribose et un phosphate qui font le lien entre les deux plateaux de bases.

Les rayonnements ionisants de faible transfert linéique d'énergie, tels que les rayons $\mathrm{X}$ et les rayons $\gamma$ du cobalt 60 , ont été étudiés d'une manière intensive. Ces rayonnements induisent principalement trois types de lésions. Il y a tout d'abord des lésions directes par rupture de la simple chaîne d'ADN, des lésions indirectes avec modification des bases, et enfin des ruptures de la double chaîne d'ADN.

Je vais détailler la nature de ces trois types de lésions. Tout d'abord, la rupture "simple chaîne » se traduit expérimentalement par une fragmentation de l'ADN, que l'on détecte par une diminution de sa viscosité quand il est extrait de cellules irradiées, et aussi par une diminution de son poids moléculaire tel qu'on peut le mesurer dans un gradient de sucrose alcalin; cette méthode permet de dissocier les deux brins d'ADN et de mesurer le poids moléculaire des brins. On constate une telle diminution du poids moléculaire de l'ADN irradié in vitro, et également si on a extrait d'une manière très douce l'ADN de cellules irradiées. Comment s'effectue cette rupture " simple chaîne "?

De très nombreux travaux ont été consacrés à ce sujet au cours des cinq dernières années. Les ruptures peuvent s'effectuer soit au niveau du sucre, c'est-à-dire du désoxyribose, et c'est essentiellement la liaison $3^{\prime}-4^{\prime}$ qui est rompue par les rayonnements de faible transfert linéique d'énergie. Il existe également des ruptures "simple chaîne " qui se font entre le phosphate et le sucre et dans ce cas, ce sont essentiellement les terminaisons $3^{\prime} \mathrm{OH}-5^{\prime}$ phosphate qui sont rompues. Le nombre des ruptures de la liaison phosphodiester ou au niveau du sucre est réduit en anoxie. On a trouvé qu'en présence d'oxygène, on augmentait la proportion de ces ruptures "simple chaîne » et ceci est évocateur de l'effet oxygène que nous connaissons au niveau de la survie cellulaire ou de l'induction des effets génétiques.

Le deuxième type de lésions est constitué par les dommages des bases; il s'avère que des quatre bases de l'ADN, la plus radiosensible est la thymine. On a identifié jusqu'ici plus d'une vingtaine de radioproduits de la thymine, ceci à la suite d'études faites in vitro sur les bases libres ou sur les nucléotides ou les nucléosides en solution. Dans les cellules ou les virus irradiés, on a identifié un de ces produits d'une manière très nette, il s'agit de l'hydroperoxyde de thymine; ce sont essentiellement les radicaux $\mathrm{OH}$ radioformés qui sont responsables d'un effet indirect sur cette base. On a également identifié des radioproduits de la cytosine in vitro; ils sont formés en proportion bien moindre que les dérivés de la thymine. 
Le troisième type de lésions déjà mentionné est la rupture « double chaîne ». Il s'agit d'une observation expérimentale; on peut discuter du mode de production de ces ruptures, mais il est clair que lorsqu'on irradie des cellules de mammifères ou des micro-organismes et, qu'ensuite, on lyse doucement les cellules sur un gradient de sucrose neutre pour ne pas dissocier les ponts hydrogène qui font la jointure entre les deux brins de l'ADN, on constate une diminution du poids moléculaire de l'ADN qui s'explique par la rupture de la double chaîne.

Passons maintenant à l'aspect plus quantitatif qui nous intéresse en tant que radioprotectionnistes; quelle est la proportion relative de ces trois types de lésions? Pour une dose de 10 rads, il se forme par cellule en moyenne 100 ruptures "simple chaîne", 10 ruptures "double chaîne" et 50 à 150 dommages de bases (essentiellement, hydroperoxyde de thymine). Ces données sont, en fait, extrapolées de données expérimentales obtenues pour des doses réelles de l'ordre de 150 rads; c'est-à-dire qu'on peut déduire le nombre de ces événements pour un rad en supposant qu'aux faibles doses il se forme la même proportion de lésions.

Or, on constate que pour une telle dose de 10 rads, dans le cas par exemple de cellules de mammifères en culture, $90 \mathrm{p}$. cent de la population, sinon plus, survit très bien. Cela peut signifier que ces lésions sont en partie ignorées par la cellule; cette possibilité n'est pas exclue. En effet, la biochimie et la génétique nous montrent qu'une fraction relativement très faible du génome d'une cellule eucaryote sert d'un point de vue informationnel; il y a énormément de redondances, de répétitions dans l'ADN des cellules eucaryotes; on estime qu'il y a environ 10 à 20 p. cent de cet $A D N$ qui, d'un point de vue génétique, est important. Une deuxième alternative paraît pour l'instant plus plausible; elle s'appuie sur des arguments expérimentaux, et fait intervenir la notion de réparation. Ainsi, une fraction très importante de ces lésions serait réparée et la survie reste très élevée.

Quels sont les arguments qui sont en faveur de la réparation ? Il y a tout d'abord des études qui ont été faites sur les micro-organismes. En effet, dès les années 1956-1957, on savait qu'à côté des formes sauvages d'Escherichia coli, il existait des mutants extrêmement radiosensibles (20 à 50 fois plus radiosensibles que les types sauvages). Ce caractère de radiosensibilité est génétiquement transmissible. Depuis, de tels mutants ont été isolés à partir de cellules eucaryotes, de levures, de neurospora, de divers types de champignons. Plus récemment, il a été démontré que certaines affections héréditaires chez l'homme s'accompagnent d'une radiosensibilité cellulaire liée à une défectivité des systèmes de réparation.

Ceci était un premier élément qui permettait de savoir qu'il se passe quelque chose dans la souche de type sauvage, qui intervient dans la réparation d'une partie des dommages. D'autre part, on savait depuis fort longtemps que le fractionnement des doses conduisait à une amélioration de la survie.

La notion de lésions sublétales est née de ce type d'expériences. Elles ont conduit à soupçonner que, dans l'intervalle de deux doses, il se passait des événements cellulaires qui permettaient de réparer une fraction des radio- 
lésions induites. D'autre part, HAHN a montré qu'il y avait une corrélation entre cette aptitude à réparer et la forme sigmoïde de la courbe de survie. En revanche, si la courbe de survie est une exponentielle, le fractionnement de dose n'entraîne pas d'amélioration de la survie. Dans le cas décrit par HAHN, il s'agit de cellules d'hamsters chinois en culture; selon la phase physiologique de croissance, ces cellules présentent des courbes de survie différentes (cités dans [1]).

Ainsi, quand on fractionne la dose pour des cellules en phase exponentielle, on améliore la survie. Par contre, le fractionnement de dose pour des cellules en phase stationnaire, n'améliore pas la survie. L'intervalle entre les irradiations est de $6 \mathrm{~h}$ pour ces expériences. Il y a là un point intéressant en relation avec le problème des très faibles doses, car le fait de ne pas pouvoir par le fractionnement entraîner une restauration, donne un argument autorisant à extrapoler au zéro.

Pour les radiobiologistes dits fondamentalistes et les radiothérapeutes, un cas important est celui des neutrons; M. Dutreix nous a montré des expériences où les courbes de survie sigmoïdes pour des radiations de faible transfert linéique d'énergie deviennent des exponentielles pour les neutrons [4]. Il apparaît très important de vérifier qu'on n'améliore pas la survie par le fractionnement dans le cas des neutrons où, précisément, les courbes de survie apparaissent comme étant exponentielles.

J'en viens maintenant à la nature proprement dite de cette réparation.

Nous savons, d'après les données obtenues sur des micro-organismes qui se prêtent à l'analyse génétique, que les processus de réparation sont contrôlés génétiquement, et il a été possible de démontrer qu'un très grand nombre de gènes participent à cette réparation.

Par exemple, dans le cas des levures, organismes unicellulaires eucaryotes, on a identifié plus d'une douzaine de gènes indépendants qui gouvernent la sensibilité aux rayonnements ionisants. Il y a plus de 22 gènes qui contrôlent la réparation des radiolésions induites par les rayons ultraviolets. Nous sommes donc en présence, sur le plan génétique, d'un système extrêmement complexe. Voyons maintenant quels sont les processus enzymatiques mis en œuvre par ces systèmes génétiquement contrôlés. Parlons tout d'abord de la réparation de la rupture "simple chaîne ". Il a été montré qu'il existe deux types de rupture "simple chaîne ", et nous devons essentiellement à K. SMITH la notion de rupture propre et de rupture sale (cité dans [2]). La rupture propre c'est la rupture qui libère les terminaisons directement accessibles à une enzyme qu'on appelle la ligase. La ligase est une enzyme bien caractérisée (que l'on trouve aussi chez les mammifères) qui fait la liaison entre les petits segments d'ADN (l'ADN n'étant pas synthétisé de manière continue, mais par petits fragments). C'est un processus naturel au cours de la replication. A la suite d'une irradiation $\mathrm{X}$ par exemple, on provoque des ruptures et la ligase fait la jointure si le site libéré est un site naturel.

Une bonne proportion des ruptures «simple chaîne » induites par les radiations sont propres et la réparation s'effectue dans les 2 min qui suivent l'irradiation. C'est une réparation extrêmement rapide. 
Il existe une réparation plus lente de ces ruptures qui dure de 3 à $6 \mathrm{~h}$, c'est-à-dire des temps nécessaires pour que l'effet du fractionnement de dose sur la survie soit maximal. Cette réparation lente résulte du nettoyage des ruptures dites sales. Si les ruptures ne libèrent pas les terminaisons adéquates pour être reconnues par la ligase, un nouveau processus enzymatique de nettoyage intervient grâce à une nouvelle enzyme : l'exonucléase qui va digérer la séquence voisine de la rupture sale. Il va se former une brèche. Puis le brin d'ADN homologue va être pris comme matrice pour resynthétiser une séquence normale, qui sera finalement scellée par la ligase. Il y a donc une exonucléase puis une $\mathrm{ADN}$ polymérase pour remplir la brèche et, enfin, la ligase pour sceller ce segment d'ADN réparé.

En ce qui concerne les ruptures «double brin », il a été tout à l'heure soulevé le problème de l'impossibilité physique d'effectuer d'un seul coup avec des rayonnements de faible transfert linéique d'énergie, une rupture qui affecte les deux brins homologues. Or, cette brèche, que nous avons décrite comme lésion transitoire, peut couvrir facilement une dizaine de nucléotides et nous permet d'envisager de quelle manière des ruptures "double chaîne " peuvent se produire. En effet, supposons que nous ayons deux ruptures en deux sites différents non homologues : le processus de digestion dû à l'exonucléase peut aller suffisamment loin, 5 à 6 nucléotides par exemple, et il y aura un moment, de par la lenteur du processus de réparation, où la brèche va être homologue. C'est là une des manières dont on envisage la formation des ruptures "double chaîne »; il ne s'agit pas, en réalité, de l'événement primaire dans les premières microsecondes qui suivent l'irradiation, mais de ce qui se produit pendant le temps de latence nécessaire au processus de réparation. Ainsi, les processus de réparation peuvent manquer leur vocation de restaurer une structure intacte et fonctionnelle, et aboutir dans certains cas à une aggravation de la lésion; c'est là, je crois, une notion assez importante.

Nous savons de quelle manière les ruptures "simple chaine " peuvent se réparer. Comment s'effectue la réparation des lésions des bases ? C'est un processus analogue à celui que nous avons vu. Mais il faut envisager, en plus de ces étapes citées, une étape en amont : une nouvelle enzyme, l'endonucléase, reconnaît cette altération au niveau de la base et va couper l'ADN, déterminant ainsi l'étape suivante qui est la formation d'un trou. Ensuite, le processus est tout à fait analogue à celui vu tout à l'heure, c'est-à-dire qu'une exonucléase à nouveau va nettoyer ce fragment, puis l'ADN polymérase va permettre de remplir la brèche et, enfin, la ligase va effectuer la jointure et nous revenons ainsi à la structure initiale.

Pour les rayonnements ionisants, on a identifié tout récemment une enzyme qui reconnaît spécifiquement ces altérations des bases. Les principales lésions induites par les ultraviolets sont des dimères de pyrimidine. Il existe, dans ce cas également, une endonucléase, l'ultraviolet-endonucléase qui reconnaît spécifiquement ces dimères de pyrimidine. Les mutants radiosensibles d'Escherichia coli sont bloqués à l'une ou l'autre de ces étapes du processus de réparation; le blocage à l'une de ces étapes entraîne une radiosensibilité plus grande. 
L'ADN étant la cible fondamentale, le fait que la restitution d'une structure intacte s'accompagne de la restitution de la survie est d'une grande importance biologique.

Pour en terminer, la réparation de la rupture "double chaîne » est encore un sujet de controverse. Que la rupture "double chaîne » soit un événement primaire, ou qu'elle soit consécutive à la mise en œuvre des processus de réparation dont on vient de parler, il n'existe pas de démonstration expérimentale définitive d'une possibilité de réparation qui se traduirait, par exemple, par un retour au poids moléculaire normal de l'ADN mesuré dans un gradient de sucrose neutre.

Quant à la relation qu'il y a entre les phénomènes que nous venons d'étudier et la radiosensibilité, je citerai une observation due à LOHMAN concernant le cycle cellulaire; c'est un des plus anciens problèmes de la radiobiologie : pourquoi, au cours du cycle cellulaire, voit-on varier la sensibilité aux radiations, et ceci d'une manière périodique ? M. Tubiana et son équipe à Villejuif ont d'ailleurs beaucoup contribué à l'analyse de ce processus.

LOHMAN a montré que la proportion des ruptures provoquées par les rayonnements ionisants variait au cours du cycle cellulaire; les phases les plus radiosensibles sont la phase $G_{1}$ et le début de la phase $S$. Parallèlement à la diminution de la survie, on note pour ces phases une proportion plus élevée de ruptures que pour la phase $\mathrm{G}_{2}$, où les cellules sont les plus radiorésistantes (cité dans [2]).

Dans notre équipe, à Orsay, nous travaillons sur des levures. Des mutants, bloqués à différentes étapes de la réparation, ont été isolés et nous avons des données qui incitent à penser que ces variations cycliques de la radiosensibilité sont, en partie, liées à la mise en œuvre ou au blocage, selon les étapes du cycle, de ces processus enzymatiques de réparation.

Si j'avais eu le temps, je vous aurais parlé aussi des liens entre les processus cellulaires de la sénescence et de la réparation, mais peut-être ce point sera-t-il soulevé dans la discussion.

Le lecteur trouvera dans les références $[1,2,3]$ les détails expérimentaux concernant l'ensemble des travaux décrits dans cet exposé général.

\section{BIBLIOGRAPHIE}

[1] Advances in radiation biology. (Y. T. Lett, H. Adlar, M. Zelle, Eds.), Academic Press, New York, 1974, 4.

[2] Current topics in radiation research. (M. Ebert and A. Howard, Eds.), North Holland, Elsevier, Amsterdam, 1974, 7.

[3] Molecular mechanisms for repair of DNA. (P. Hanawalt and R. Setlow, Eds.), Plenum Press, New York, 1975.

[4] Dutreix A., Forme initiale de la courbe de survie des cellules irradiées, Radioprotection $1975,11,19-34$. 